\title{
Effects of Vitamin K1 Supplementation on the Risk Factors to the Stroke and on Memory in Spontaneously Hypertensive Rats Stroke Prone (SHR-sp)
}

\author{
Victor Agati Cavargere ${ }^{2}$, Marcela Rodrigues Moreira Guimarães ${ }^{3}$, Leonardo Borges \\ Murad $^{3}$, Amanda Chaves ${ }^{4}$, Lucia Marques Vianna ${ }^{1 *}$ \\ ${ }^{1}$ Head of Laboratory of Nutritional Investigation and Degenerative-Chronic Diseases. (LINDCD) - Universidade \\ Federal do Rio de Janeiro - UNIRIO. ${ }^{2}$ Msc in Neurology - Universidade Federal do Rio de Janeiro - UNIRIO. \\ Affiliated to Laboratory of Nutritional Investigation and Degenerative-Chronic Diseases (LINDCD). ${ }^{3}$ Dsc in \\ Neurology - Universidade Federal do Rio de Janeiro - UNIRIO. Affiliated to Laboratory of Nutritional \\ Investigation and Degenerative-Chronic Diseases (LINDCD). ${ }^{4}$ Universidade Federal do Rio de Janeiro - UNIRIO. \\ Affiliated to Laboratory of Nutritional Investigation and Degenerative-Chronic Diseases (LINDCD)
}

\begin{abstract}
A number of risk factors have been associated to the stroke and many strategies have been proposed in order to control them as well. Vitamin $K$ has been largely found in brain, which suggests a possible function at that tissue. This study aimed to evaluate the potential of this vitamin on the prevention of risk factors to stroke and on cognitive function on SHRSP rats. Twelve SHRSP males, 15 weeks old, were divided into two groups $(n=6$ each), receiving the vehicle-coconut oil (control group) or $40 \mu \mathrm{g}$ of phylloquinone (treated group) during 28 days. Biological parameters, systolic blood pressure and lipid profile were evaluated. Both groups were submitted to the neurological tasks. The data was treated by Student's $t$ test and ANOVA one-way test being P<0.05 considered significant. The phylloquinone supplementation showed a statistically significant reduction in the treated group of all parameters of lipid profile and systolic blood pressure when compared to the control group. Neurological evaluation indicated a statistically significant improvement in the performance of long term memory tests in the treated group, without similar findings in the evaluation of short memory. In sum, phylloquinone supplementation was shown to modulated lipid profile and protect neuronal suffering in this model.
\end{abstract}

Key words: Phylloquinone, memory, stroke, SHRSP

*Authors for correspondence: lindcd@ig.com.br 


\section{INTRODUCTION}

Cerebrovascular diseases are the second leading cause of death and dementia, and the leading cause of disability worldwide (Soler and Ruiz 2010). Studies indicated that the most commonly involved functions in cerebrovascular lesions appear to be those associated with memory and executive function (Vicario et al. 2011).

In order to minimize the incidence of stroke and its comorbidities, one should aim at controlling their risk factors. Among them, hypertension can be highlighted as the most important modifiable risk factor for any type of stroke (hemorrhagic and ischemic) (Soler and Ruiz 2010).

Apart from hypertension, there is also strong evidence for the involvement of oxidative stress in the pathogenesis of vascular disorders (Pawlak et al. 2005), working independently or associated to the dyslipidemia (Moreira et al. 2006). An increased generation of reactive oxygen species inhibits endogenous antioxidant defenses, pointing to a subsequent oxidative damage and cell death (Pong 2003). Interestingly, some evidence has shown the beneficial effects of an antioxidant nutrients in brain ischemia (Ikeda and Miyahara 2003; Murad et al. 2013).

In addition, several studies have reported significant benefits in reducing the risk of stroke and post-stroke recovery in patients who consumed foods rich in micronutrients, including $\mathrm{B}$ vitamins and antioxidant vitamins $\mathrm{E}$ and $\mathrm{C}$ (Sanchez-Moreno et al. 2009).

In this context, the phylloquinone (vitamin K1) has been highlighted by demonstrating an antioxidant effect (Tirapelli et al. 2008b; Sogabe et al. 2011). However, such findings were obtained on normotensive strains without previous vascular disease or hypertension. At the same way, the possible effect of vitamin $\mathrm{K}$ on lipid profile modulation stills controversial (Kolahi et al. 2015).

For this reason, the present study aimed to evaluate the effects of vitamin K1 supplementation on the prevention of risk factors to stroke and on cognitive function on SHRSP rats, a strain widely used as a severe arterial hypertension, stroke and memory dysfunction model.

\section{MATERIALS AND METHODS}

\section{Animals}

Male, 15-week-old stroke-prone spontaneously hypertensive rats, SHRSP $(n=12)$ were obtained from the colony of rats in the bioterium of the Federal University of Rio de Janeiro State. The rats were maintained in metabolic cages with controlled conditions of temperature $\left(21 \pm 2{ }^{\circ} \mathrm{C}\right)$, humidity $(60 \pm 10 \%), 12 \mathrm{~h}$ dark/light cycle (exposed to artificial light from 7:00 am to 7:00 $\mathrm{pm})$ and air ventilation $(15 \mathrm{~min} / \mathrm{h})$. The rats received rat chow food pellets (Nuvilab from Nuvital Co, PR, Brazil) and water ad libitum. All procedures were carried out in accordance with the conventional guidelines for experimentation with animals (National Institute of Health, Publication no. 85-23, revised 1996). The Ethics Committee for Animal Experimentation at the Federal State University of Rio de Janeiro approved the experimental protocol used in this study.

\section{Groups and supplementation}

Initially, the rats were maintained under basal conditions for 14 days. After, they were subdivided into two groups $(\mathrm{n}=6$ each) and were treated as follows: control group- received the vehicle coconut oil (Sigma-Aldrich, C-1758, St. Louis, MO) $(0.3 \mathrm{~mL})$ and treated group was treated with $40 \mu \mathrm{g}$ of phylloquinone (SigmaAldrich, 47773, St. Louis, MO) diluted in $0.3 \mathrm{~mL}$ of coconut oil. Both groups received daily doses of the vehicle or vitamin by oral gavage using a polyethylene catheter PE 190, during 28 days.

\section{Physiologic parameters and blood pressure}

The body weight, diuresis, food and water intake were measured daily and systolic blood pressure was determined by plethysmography twice a week.

\section{MEMORY AND COGNITION TEST}

\section{Morris water maze}

The Morris Water Maze measured $1.8 \mathrm{~m}$ in diameter and was $60 \mathrm{~cm}$ deep. Before filling it with water we added an escape platform affixed in a permanent location in the maze. Next we filled the apparatus halfway with water plus milk powder, to a depth of $30 \mathrm{~cm}$. Thus, the hidden platform remained a few mm below the surface of the water. SHRSP were placed in the Morris Water Maze, and when released, the animals swam 
around the pool in search of an escape (hidden platform). The time it took the animals to find the platform was measured in seconds. The test was conducted twice daily for each rat.

\section{Novel object recognition test}

Initially, each SHRSP was placed in a plastic box and introduced to two differently-shaped objects to explore freely for five min. This procedure was repeated after $180 \mathrm{~min}$. Additionally, $180 \mathrm{~min}$ after the last exercise, one of the objects was replaced by a new object with a different shape. We thus studied the time the rat spent with the new object and with the familiar object. Each test lasted 10 min. In this step we studied the preservation of short-term memory.

To assess the preservation of long-term memory, twenty-four $\mathrm{h}$ after the last test, the novel object from the previous day was replaced by another. The time spent with this novel object and the familiar object also was recorded. These tests lasted $10 \mathrm{~min}$.

For the short- and long-term-memory procedures, exploration was defined as sniffing or touching the object with the nose or front leg. When an animal finished the test and another was to be introduced, the objects were cleaned with alcohol and dried with paper towels, to prevent a bias in the results due to olfactory perception. These tests were applied during the basal period and twice a week over the course of the entire experiment.

\section{Sacrifice}

The control and treated groups were anesthetized with sodium pentobarbital intraperitoneally (25 $\mathrm{mg} / \mathrm{kg}$ ) and sacrificed by heart puncture. Blood was collected from each rat and placed in a tube, and the tubes were then centrifuged at 2000 RPM $\mathrm{x} g$ for $10 \mathrm{~min}$ to obtain the serum.

\section{Lipid profile}

Total cholesterol (TC), HDL-cholesterol and triglycerides (TG) were measured by an enzymatic assay. LDL-cholesterol levels were calculated by the Friedewald formula: $\mathrm{LDL}=\mathrm{TC}-\mathrm{HDL}-$ (TG/5).

\section{Statistical analysis}

The data were reported as the mean and standard deviation (SD) and subsequently analyzed using the Student's t test to compare control group with treated group and ANOVA one-way test to evaluate the evolution of the groups during the weeks of the experiment. $\mathrm{P}$ value of $<0.05$ was considered significant. The statistical software package used was GraphPad Prism ${ }^{\circledR} 5.0$ for Windows ${ }^{\circledR}$ (Graph Pad Software, San Diego, CA, USA).

\section{RESULTS}

All the animals on the experiment showed variation on body weight and other general physiologic parameters during the study. However, there was no statistical significance (Table 1).

Table 1- The data represents Mean \pm SD of body weight, urine output, water intake and diet of control group ( $n=6)$ and treated group of SHRSP rats $(n=6)$.

\begin{tabular}{l|l|l|l|l|l|l}
\hline Parametes & Groups & Basal Period & $1^{\mathrm{a}}$ Week & $2^{\mathrm{a}}$ Week & $3^{\mathrm{a}}$ Week & $4^{\mathrm{a}}$ Week \\
\hline Body weight & Control & $197.33 \pm 13.74$ & $201323 \pm 16.83$ & $210.87 \pm 7.84$ & $218 \pm 10.55$ & $216.97 \pm 9.86$ \\
$(\mathrm{~g})$ & Treated & $195.57 \pm 9.11$ & $201.47 \pm 10.63$ & $195.2 \pm 16.33$ & $217.21 \pm 18.68$ & $219.93 \pm 19.69$ \\
\hline Urine Output & Control & $9.87 \pm 3.43$ & $5.63 \pm 2.03$ & $6.54 \pm 2.33$ & $6.45 \pm 2.33$ & $7.83 \pm 1.23$ \\
$(\mathrm{~mL})$ & Treated & $6.78 \pm 1.5$ & $9.96 \pm 4.28$ & $9.86 \pm 9.38$ & $8.03 \pm 3.55$ & $8.66 \pm 5.57$ \\
\hline Diet $(\mathrm{g})$ & Control & $14.56 \pm 2.32$ & $15.47 \pm 1.34$ & $15.99 \pm 0.87$ & $13.57 \pm 2.07$ & $17.56 \pm 3.36$ \\
& Treated & $19.99 \pm 2.08$ & $14.85 \pm 0.85$ & $15.15 \pm 1.84$ & $19.66 \pm 0.97$ & $21.10 \pm 1.80$ \\
\hline Water Intake & Control & $45.98 \pm 3.46$ & $49.69 \pm 4.76$ & $37 \pm 6.68$ & $32.68 \pm 3.23$ & $38.67 \pm 5.78$ \\
$(\mathrm{~mL})$ & Treated & $53.05 \pm 4.34$ & $52.38 \pm 29.8$ & $49 \pm 9.40$ & $49.9 \pm 9.83$ & $35.6 \pm 8.38$ \\
\hline
\end{tabular}

Regarding animals ectoscopy, no change in the skin, mucosa, or behavioral changes were observed in any of the groups.

Regarding lipid profile, total cholesterol of the treated group was significantly lower than the control group. In other lipid fractions the trend remained, and a statistically significant reduction in all the evaluated data was observed (Fig. 1). 


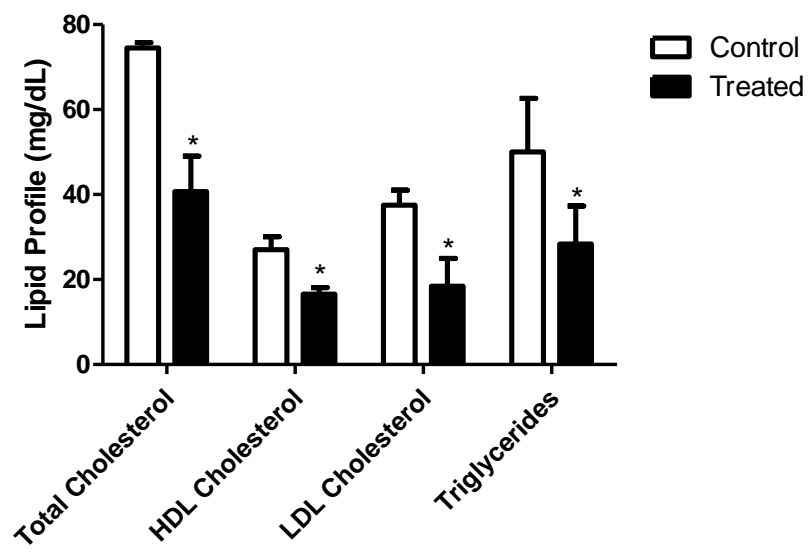

Figure 1- The data represents mean \pm SD of lipid profile on control group $(n=6)$ and treated group of SHRSP rats $(n=6)$.

$* \mathrm{p}<0.05$ when compared with control group.

Regarding the blood pressure of the animals, both groups had similar values at the beginning of the study (basal period and first week of supplementation) as expected from a random population. However, at the second week there was a significant $(p<0.05)$ reduction of the systolic blood pressure in the treated group, which intensified by the end of the experiment (3rd and $4^{\text {th }}$ week) (Fig. 2).

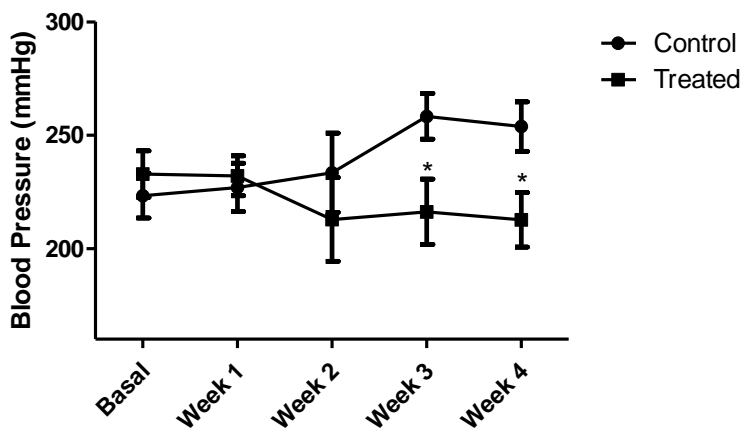

Figure 2- The data represents mean \pm SD values of the systolic blood pressure during the period of the experiment, between control group $(n=6)$ and treated group of SHRSP rats $(n=6)$.

$* p<0.05$ when compared with control group.

Regarding memory, two parameters were checked to assess the short term memory of the animals. Figure 3, shows the mean \pm SD of Time Spent with Novel Object (TSNO) in both groups. The variations found in this parameter during the experiment were not statistically significant $(\mathrm{p}=$
0.072). In the following graph, the same representation illustrating Time Spent with Family Object (TSFO), like the last, statistical analysis was not significant $(\mathrm{p}=0.071)$, showing that the short memory of the animals was not altered by vitamin supplementation (Fig. 4).

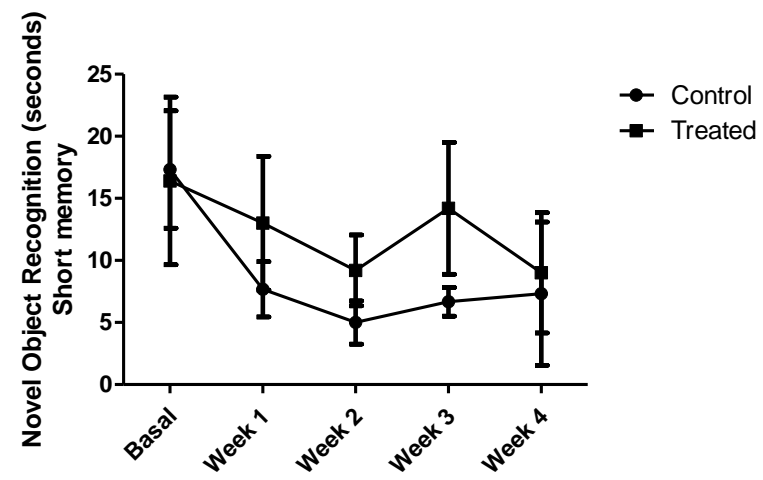

Figure 3- The data represents the results of time taken to the recognition of novel object between control group $(n=6)$ and treated group of SHRSP rats $(n=6)$. Short term memory. 


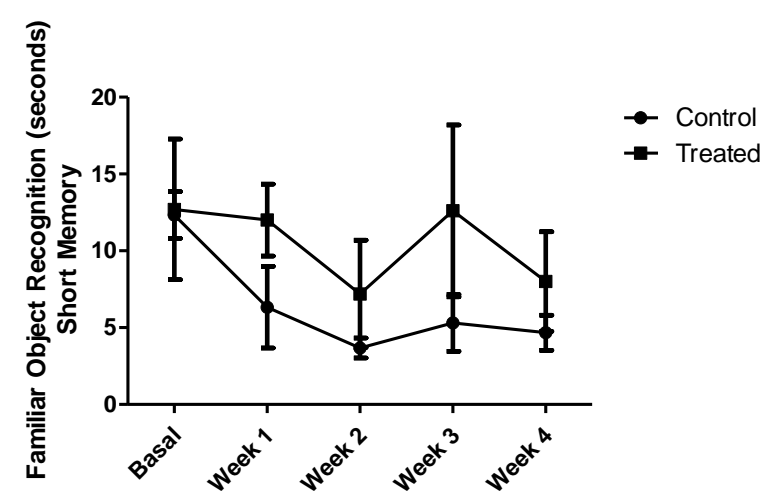

Figure 4 - The data represents the results of time taken to the recognition of familiar object between control group $(n=6)$ and treated group of SHRSP rats $(n=6)$. Short term memory.

As in the short memory, long memory assessment was done through the parameters of TSNO and TSFO. Figure 5 shows the gap between mean values $\pm \mathrm{SD}$ increasing throughout the experiment, resulting in a statistically significant difference.

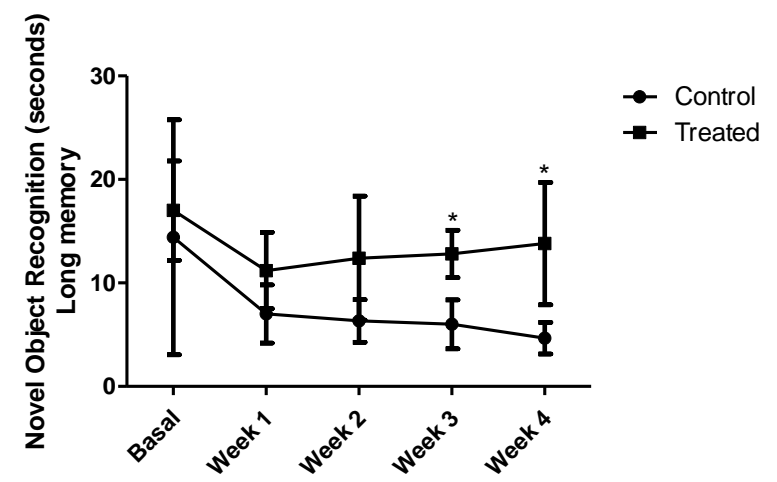

Figure 5 - The data represents the results of time taken to the recognition of novel object between control group $(n=6)$ and treated group of SHRSP rats $(n=6)$. Long term memory.

$* \mathrm{p}<0.05$ when compared with control group.

Following this trend, the treated group spent less time than the control group with the familiar object, while the latter group remained almost constant in this parameter, setting a statistically significant difference between them (Fig. 6).

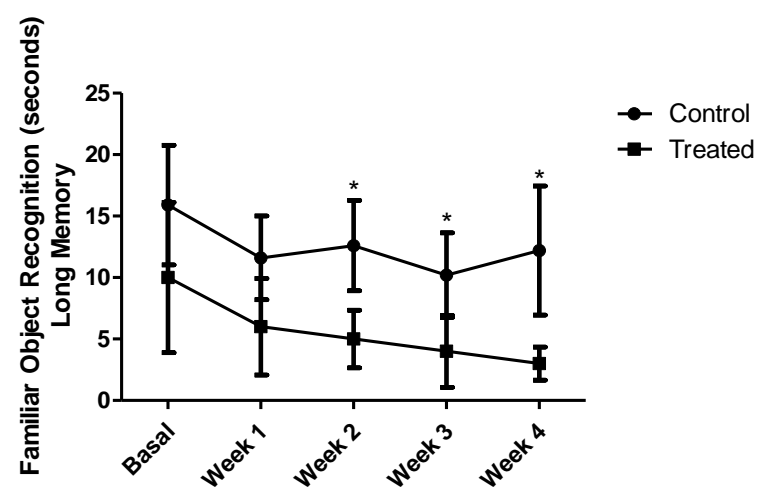

Figure 6 - The data represents the results of time taken to the recognition of familiar object between control group $(n=6)$ and treated group of SHRSP rats $(n=6)$. Long term memory.

${ }^{*} \mathrm{p}<0.05$ when compared with control group.

With regard to standards of maintenance or loss of memory function, the maze test showed a significant reduction in the execution time of the task in the treated group when compared to the control group (Fig.7).

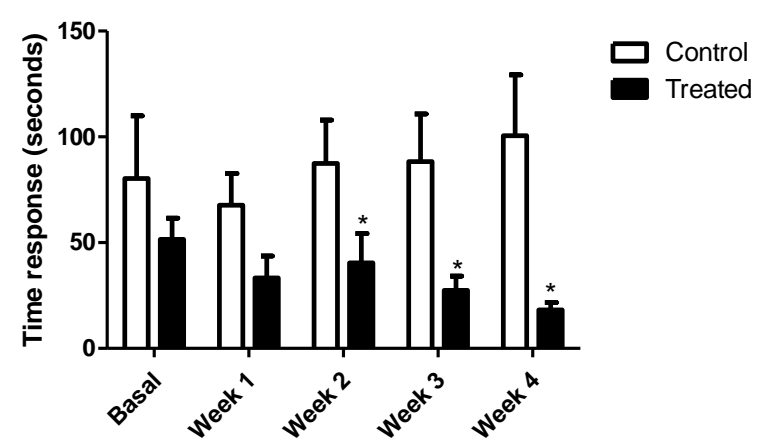

Figure 7- The data represents the results of time taken to complete the maze test between control group $(n=6)$ and treated group of SHRSP rats $(n=6)$.

$* \mathrm{p}<0.05$ when compared with control group.

The results of the Morris water maze confirm the results above. While the control group showed an increase in the execution time of the test, treated animals showed a progressive decrease in the same parameter (Fig. 8). 


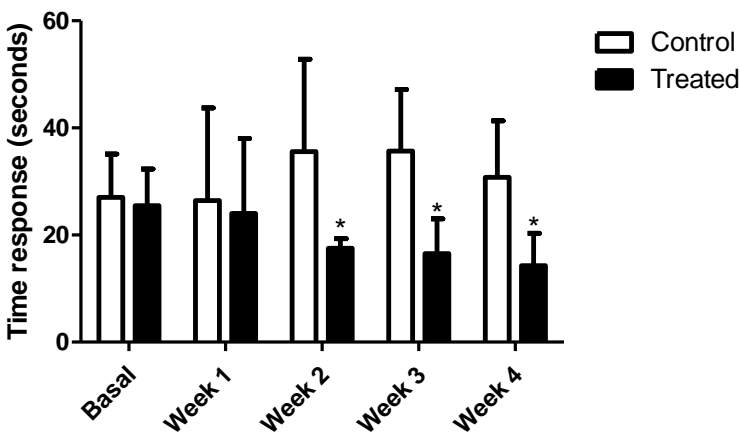

Figure 8 - The data represents the results of time taken to find the hidden platform on Morris Water Maze Test between control group $(\mathrm{n}=6)$ and treated group of SHRSP rats $(n=6)$.

$* \mathrm{p}<0.05$ when compared with control group.

\section{DISCUSSION}

The present work demonstrates that SHRSP rats were responsive to the supplemental phylloquinone, mainly regarding the dyslipidemia and hypertension attenuation, intrinsic characteristics of this strain, and reveals that there were no apparent signs of nutritional interaction towards the use of supraphysiological doses.

The results presented here confirm previous findings when vitamin $\mathrm{K} 1$ was administered intravenously (Tirapelli et al. 2002a; Tirapelli et al. 2008b). The first proposes, as hypotensive mechanism, that the phylloquinone prevents carotid artery contraction induced by the action of phenylephrine during hypoxia in rats. The latter suggests that the effect on blood pressure reduction of this vitamin involves the generation of nitric oxide (NO) and vasodilatory prostaglandins, since the concomitant administration of L-NAME and indomethacin were able to mitigate and/or inhibit the hypotensive effect of vitamin K1.

In fact, the literature present others pausible mechanisms associated to the hypotensive effect of vitamin K. According to Luo et al (1997), phylloquinone is an essential cofactor of the matrix (MGP) GLA protein, whose function is to inhibit arterial calcification. Thus, vitamin K1 supplementation could stimulates MGP action, allowing the proper functioning of the endothelium and its regulatory activities on blood pressure. In addition, Price et al. (1998) observed an arterial calcification of knockout mice for MGP producing gene. Essalihi et al. (2003) by using warfarin, a known vitamin $\mathrm{K}$ antagonist, observed an important hypertension and arterial calcification as well.

Another alternative, which does not exclude the previously mentioned, could be through the reduction of all lipid fractions as it was reached in the present report. According to two independent studies (O'donnell et al. 2010; Ginsberg 2013), the reduction of lipid fractions is beneficial in controlling hypertension, because hyperlipidemia is the most harmful factor for the endothelium. With endothelial injury, there is the onset of the inflammatory process with the expression of adhesins, integrins and production of chemokines. Consequently, there is a migration of monocytes and $\mathrm{T}$ lymphocytes, cronificating local inflammation by stimulating the smooth muscle cells and extracellular matrix synthesis.

The transport of vitamin $\mathrm{K}$ in the blood is closely associated with triglyceride and cholesterol-rich lipoproteins as it is a highly lipophilic substance, binding, almost exclusively, with lipoproteins such as LDL, VLDL and chylomicrons (Kohlmeier et al. 1996).

In an enlightening study, after oral consumption, traced phylloquinone was absorbed in the duodenum and was associated with chylomicrons. The rate of clearance from the blood of both the phylloquinone and chylomicrons was measured and it was found that both were removed from circulation at the same rate. Knowing that these lipoproteins are responsible for more than half of this vitamin entrainment, the findings suggest that the stimulus for the removal of phylloquinone is also responsible for the reduction of lipid fractions (Ichihashi et al. 1992).

Regarding to the effects of phylloquinone supplementation on the neurological response, the sensory-motor tests revealed no statistical difference between the treated and control groups in the present assay; however memory functioning was significantly improved by this vitamin. Carrié et al. (2004), also suggested that vitamin K1 has a crucial role on memory function in young rats, which was attributed to it action on sphingolipids metabolism. According to those authors, vitamin $\mathrm{K}$ supplementation promoted a decrease of ceramide brain levels in the hippocampal region.

Data from in vivo and in vitro studies suggest that the role of vitamin $\mathrm{K}$ is in the regulation of many enzymes involved in the metabolism of sphingolipids in myelin-rich regions of the brain (Denisova and Booth 2005), mainly in the medulla, pons, and midbrain, where there are 
higher concentration of this vitamin (Carrié et al. 2004).

It has been shown that ceramides act in processes of cellular growth, division, differentiation and apoptosis (Zeidan and Hannun 2007), but, when present in high concentrations, are strongly related to the inflammatory processes (Ballou et al. 1996) and to the generation of reactive oxygen species by mitochondria (Jana et al. 2009). In addition, several studies have reported the relationship between high concentrations of ceramides and neurodegenerative disorders such as seen in Azheimer's disease (He et al. 2010).

For fixation of long term memory, the stimulus must pass a neural circuit called Papez Circuit which includes the hippocampal neurons - as well as generate new synapses and anatomical changes, while the short memory requires only the maintenance of certain proteins and neurotransmitters in the synaptic space (Kandel, 2001). It is known that one of the most sensitive brain sites to hypoxia is the CA1 region of the hippocampus (Schunke et al. 2007), and a lesion in this circuit impairs the establishment of new memories (Bussey et al. 1999). In addition, SHRSP rats, at higher oxidative stress characteristic of this strain, are under greater influence of neuronal damage, which increases ceramide levels and impairs the occurrence of necessary changes in hippocampal cells for the consolidation of memory. Phylloquinone supplementation, as discussed above, is able to protect neurons from this suffering by keeping the cells and circuitry in better physiological conditions, which facilitates the establishment of long memory.

Although it is believed that the vitamin $\mathrm{K}$ vitamine that plays a role in brain tissue is menadione-4 (MK-4), responsible for 99\% of all vitamin K found at that location, certain tissues have the ability to convert phylloquinone to MK-4. (Crivello et al. 2010).

A clarifying experiment showed that the conversion of phylloquinone to menadione is tissue-dependent. In their study, phylloquinone was not found in the brain after the intake of that radiolabelled substance, being found only in marked MK-4. This strongly suggests that all phylloquinone is converted into MK-4 in the brain. In addition, prolonged diets with different concentrations of vitamin K1 significantly reflected such differences in brain tissues (Crivello et al. 2010).
In sum, phylloquinone supplementation of SHRSP rats was shown to modulated lipid profile and protect neuronal suffering caused by higher oxidative stress, characteristic of this strain.

\section{ACKNOWLEDGMENTS}

This work was supported by the Brazilian agencies CNPq, CAPES and UNIRIO.

\section{REFERENCES}

Ballou LR, Laulederkind SJ, Rosloniec EF, Raghow R. Ceramide signaling and the immune response. Biochim Biophys Acta. 1996;1301: 273278.

Bussey TJ, Muir JL, Aggleton JP. Functionally dissociating aspects of event memory: the effect of combined perihinal and postrhinal cortex lesions on object and place memory in the rat. J Neurosci. 1999; 1:19(1): 495-502.

Carrié I, Portoukalian J, Vicaretti R, Rochford J, Potvin $\mathrm{S}$, Ferland G. Menaquinone-4 concentration is correlated with sphingolipid concentrations in rat brain. J Nutr. 2004;134(1): 167-172.

Crivello NA, Casseus SL, Peterson JW, Smith DE, Booth SL. Age- and brain region-specific effects of dietary vitamin $\mathrm{K}$ on myelin sulfatides. $J$ Nutr Biochem. 2010; 21(11): 1083-1088.

Denisova NA, Booth SL. Vitamin K and sphingolipid metabolism: evidence to date. Nutr Rev. 2005;63(4): 111-121.

Essalihi R, Dao HH, Yamaguchi N, Moreau P. A new model of isolated systolic hypertension induced by chronic warfarin and vitamin $\mathrm{K} 1$ treatment. Am J Hypertens. 2003;16(2): 103-110.

Ginsberg H. Statins in cardiometabolic disease: what makes pitavastatin different? Cardiovasc Diabetol. 2013; 12(1): S1.

He X, Huang Y, Li B, Gong CX, Schuchman EH. Deregulation of sphingolipid metabolism in Alzheimer's disease. Neurobiol Aging. 2010;31: 398408.

Ichihashi T, Takagishi Y, Uchida K, Yamada $\mathrm{H}$. CoIonic absorption of menaquinone-4 and menaquinone-9 in rats. J Nutr. 1992;122: 506-512.

Ikeda S, Miyahara Y. Ischemic heart disease and oxidative stress. Nihon Rinsh. 2003; 61(5): 831-836.

Jana A, Hogan EL, Pahan K. Ceramide and neurodegeneration: susceptibility of neurons and oligodendrocytes to cell damage and death. J Neurol Sci. 2009; 278: 5-15.

Kandel E. The molecular biology of memory storage: a dialogue between genes and synapses. Science. 2001; 294: 1030-1038. 
Kohlmeier M, Salomon A, Saupe J, Shearer MJ. Transport of vitamin $\mathrm{K}$ to bone in humans. J Nutr. 1996;126(4): 1192S-1196S.

Kolahi S, Pourghassem Gargari B, Mesgari Abbasi M, Asghari Jafarabadi M, Ghamarzad Shishavan N. Effect of phylloquinone supplementation on lipid profile in women with rheumatoid arthritis: a double blind placebo controlled study. Nutr Res Pract. 2015; 9(2): 186-191.

Luo G, Ducy P, Mckee MD, Pinero GJ, Loyer E, Behringer RR et al. Spontaneous calcification of arteries and cartilage in mice lacking matrix GLA protein. Nature.1997;385: 78-81.

Moreira TS, Takakura AC, Sato MA, Menani JV, Colombari E. Antihypertensive responses elicited by central moxonidine in rats: possible role of nitric oxide. J Cardiovasc Pharmacol. 2006;47(6): 780-787.

Morris RGM. Developments of a water-maze procedure for studying spatial learning in the rat. J Neurosci Methods II. 1984: 47-60.

Mumby D. Object Recognition. The behavior of the laboratory rat. Oxford University Press. 2005;36: 383-391.

Murad LB, Guimarães MR, Paganelli A, Basilio-deOliveira CA, Vianna LM. Alpha-tocopherol in the brain tissue preservation of stroke-prone spontaneously hypertensive rats. J Physiol Biochem. 2013; 70 (1): 49-60.

O'donnell MJ, Xavier D, Liu L, Zhang H, Chin SL, Rao-Melacini $\mathrm{P}$ et al. Risk factors for ischaemic and intracerebral haemorrhagic strike in 22 countries (the INTERSTROKE study): a case-control study. Lancet. 2010; 10,376(9735): 112-123.

Pawlak R, Rao BS, Melchor JP, Chattarji S, McEwen B, Strickland S. Tissue plasminogen activator and plasminogen mediate stress-induced decline of neuronal and cognitive functions in the mouse hippocampus. Proc Natl Acad Sci. 2005;13,102(50): 18201-18206.

Pong K. Oxidative stress in neurodegenerative diseases: therapeutic implications for superoxide dismutase mimetics. Expert Opin Biol Ther. 2003;3(1): 127-139.
Price PA, Faus SA, Williamson MK. Warfarin causesrapid calcification of the elastic lamellae in rat arteries and heart valves. Arterioscler Thromb Vasc Biol. 1998;18: 1400-1407.

Sanchez-Moreno J, Martinez-Aran A, Colom F, Scott J, Tabares-Seisededos R, Sugranyes G et al. Neurocognitive dysfunctions in euthymic bipolar patients with and without prior history of alcohol use. J Clin Psychiatry. 2009;70(8): 1120-1127.

Schunke M, Schulte E, Schumacher U. Prometheus, atlas de anatomia: cabeça e pescoço. Rio de Janeiro: 1. ed. Guanabara Koogan, 2007.

Sogabe N, Maruyama R, Baba O, Hosoi T, GosekiSone M. Effects of long-term vitamin $\mathrm{K}_{1}$ (phylloquinone) or vitamin $\mathrm{K}_{2}$ (menaquinone-4) supplementation on body composition and serum parameters in rats. Bone. 2011;48(5): 1036-1042.

Soler EP, Ruiz VC. Epidemiology and Risk Factors of Cerebral Ischemia and Ischemic Heart. Diseases: Similarities and Differences. Curr Cardiol Rev. 2010; 6: 138-149.

Tirapelli CR, Mingatto FE, De Oliveira AM. VitaminK1 prevents the effect of hypoxia on phenylephrine-induced contraction in the carotid artery. Pharmacology. 2002a, 66: 36-43.

Tirapelli CR, Resstel LB, De Oliveira AM, Correa FM: Mechanisms underlying the biphasic effect of vitamin K1 (phylloquinone) on arterial blood pressure. $J$ Pharm Pharmacol. 2008b, 60: 889-893.

Vicario A, Del Sueldo MA, Zilberman JM, Cerezo GH: Cognitive evolution in hypertensive patients: a sixyear follow-up. Vasc Health Risk Manag. 2011, 7: 281-285.

Zeidan YH, Hannun YA. Translational aspects of sphingolipid metabolism. Trends Mol Med. 2007, 1: 327-336. 